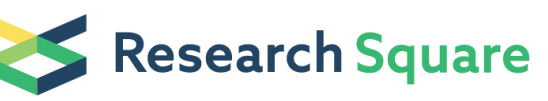 \\ Preprints are preliminary reports that have not undergone peer review. \\ They should not be considered conclusive, used to inform clinical practice, or referenced by the media as validated information.
}

\section{Analysis of Lymph Node Metastasis and Risk Factors in 975 Patients with FIGO 2009 Stage $\llbracket A-\llbracket A$ Cervical Cancer}

\section{Lina Cao}

Beijing Medical College: Capital Medical University https://orcid.org/0000-0002-9151-0959

Weimin Kong ( $\nabla$ kwm1967@ccmu.edu.cn )

Beijing Medical College: Capital Medical University https://orcid.org/0000-0002-3283-8620

Jing Li

Beijing Medical College: Capital Medical University

\section{Dan Song}

Beijing Medical College: Capital Medical University

\section{Bixia Jin}

Beijing Medical College: Capital Medical University

Tingting Liu

Beijing Medical College: Capital Medical University

\section{Chao Han}

Beijing Medical College: Capital Medical University

\section{Research Article}

Keywords: Cervical cancer, Lymph node metastasis (LNM), Distribution characteristics, Risk factors

Posted Date: January 3rd, 2022

DOI: https://doi.org/10.21203/rs.3.rs-1030472/v1

License: () (1) This work is licensed under a Creative Commons Attribution 4.0 International License. Read Full License 


\section{Abstract \\ Objective}

To summarize the lymph node metastasis (LNM) rate of patients with stage $₫ A-\llbracket A$ cervical cancer, and further analyze its distribution characteristics and related risk factors.

\section{Methods}

According to FIGO 2009, the clinical data of 975 patients with stage $₫ A-\varangle A$ cervical cancer treated in our hospital from January 2010 to December 2018 were retrospectively analyzed. The incidence and distribution of LNM were analyzed, and the influencing factors of cervical cancer LNM were analyzed by univariate and multivariate Logistic regression.

\section{Results}

In this study, the LNM rate was 14.8\%(144/975), and a total of 20288 lymph nodes were removed, among which 359 lymph nodes had metastasis. According to the statistics of the number and frequency of metastatic lymph nodes in different regions, the metastatic rate was the highest in the parauterine/obturator regions. Univariate analysis showed that pregnancy $>3$ times, tumor size $>4 \mathrm{~cm}$, gross type, FIGO stage, pathological type, positive lymphovascular space invasion (LVSI), deep cervical stromal invasion (outer $1 / 2$ invasion), parametrial involvement and uterine corpus invasion $(\mathrm{UCl})$ were all correlated with $\mathrm{LNM}(\mathrm{P}<0.05)$. Multivariate analysis showed tumor lesion >4cm (OR=2.253,95\%Cl:1.486-3.416,P<0.001), positive LVSI (OR=5.353,95\% Cl: 3.303-8.676, $\mathrm{P}<0.001)$, deep cervical stromal invasion ( $\mathrm{OR}=3.461,95 \% \mathrm{Cl}$ : 2.106-5.688, $\mathrm{P}<0.001)$ and deep UCl (myometrial invasion $\geq 50 \%$ ) $(\mathrm{OR}=3.529,95 \% \mathrm{Cl}: 1.321-9.427, \mathrm{P}=0.012)$ were independent risk factors for $L N M$.

\section{Conclusion C}

ervical cancer patients are more likely to have LNM if the tumor size $>4 \mathrm{~cm}$, positive LVSI, deep cervical stromal invasion and deep UCI. In clinical treatment, attention should be paid to the evaluation of LNM.

\section{Introduction}

Cervical cancer is the fourth most common cancer in women in the world [1, 2]. Lymph node metastasis (LNM) is the main way of cervical cancer metastasis, and also is an important factor affecting the prognosis of patients [3, 4]. The International Federation of Gynecology and Obstetrics (FIGO)2018 new staging of cervical cancer have included LNM. Once LNM occurs, staging is IIIC [5]. The revised FIGO stage reflects the importance of LNM [6], and can better assess the risk of cervical cancer patients [7].

For early-stage cervical cancer, the main purpose of lymph node resection is to determine whether there is positive lymph node, and to provide a basis for postoperative supplementary therapy. In China, sentinel lymph node biopsy is not widely used, and most early-stage cervical cancer has undergone systematic lymph node resection, which leads to many cervical cancer patients without LNM to receive unnecessary surgery. Especially for early-stage low- 
risk patients (tumor size < 2cm, no LVSI, depth of invasion < 10mm, no LNM indicated by imaging examination) [8], which leads to increased surgical risk and complications.

So which patients are more likely to have LNM? There are many studies about LNM of cervical cancer [9-14], but few studies on large samples. Since our hospital is a large obstetrics and gynecology hospital in China, many cervical cancer patients were admitted to our hospital. In this study, 975 patients with FIGO 2009 early-stage cervical cancer in our hospital were analyzed for LNM, so as to enhance the attention of preoperative evaluation of LNM and provide evidence for clinical treatment.

\section{Material And Methods}

\section{Patients}

The clinical data of cervical cancer patients admitted to Beijing Obstetrics and Gynecology Hospital affiliated to Capital Medical University/Beijing Maternal and Child Health Care Hospital from January 2010 to December 2018 were collected. Inclusion criteria were: (1)pathological diagnosis of cervical cancer, including squamous carcinoma, adenocarcinoma and adenosquamous carcinoma; (2)FIGO 2009 stage $₫ A-\varangle A$; (3)radical hysterectomy combined with pelvic lymphadenectomy and/or para-aortic lymphadenectomy; (4)complete clinical data. Exclusion criteria were: (1) cervical cancer of pregnancy; (2)cancer of the cervical stump; (3)special and rare types of cervical cancer》 (4)presence of a cancer other than cervical cancer; (5)others do not meet inclusion criteria.

A total of 975 patients were included, ranging in age from 21 to 73 years, with an average age of (45.95 \pm 9.20$)$ years; FIGO stage: $\triangle A 64$ cases, $\triangle B \quad 706$ cases, $\triangle A 205$ cases; Histological types: 697 cases of squamous carcinoma, 136 cases of adenocarcinoma, 142 cases of adenosquamous carcinoma; Para-aortic lymph nodes were resected in 58 cases without metastasis.

The patients were divided into the positive group (the metastasis group, $n=144$ ) and the negative group (the nonmetastasis group, $\mathrm{n}=831$ ) based on the postoperative pathology examination.

\section{Clinical data collection}

Bilateral pelvic lymph nodes were divided into five regions: common iliac, external iliac, internal iliac, parauterine/obturator and deep inguinal(suprafemoral) lymph nodes. The number of metastatic lymph nodes in each region was counted and the proportion was calculated. Due to the different total number of lymph nodes in each region, the distribution was again calculated according to the frequency of occurrence of metastatic lymph nodes in each region. Then age, body mass index (BMI), menopausal status, number of pregnancies and births, prior surgical history, tumor size, FIGO stage, gross type (exophytic or endophytic tumor), histological type, differentiation, stromal invasion depth, lymphovascular space invasion (LVSI), uterine corpus invasion (UCI), parametrial involvement (PMI) and vaginal margin were analyzed to explore risk factors of cervical LNM. The difference in the clinical presentation of tumors is called the gross type. Tumors were classified according to the dominant morphology for clinical presentation. Ulcerative tumors and tumors with barrel-shaped morphology were referred to as endophytic tumors. Fungating tumors with or without small superficial ulcerations were referred to as exophytic tumors.

\section{Statistical analysis}


Statistical analyses were performed using SPSS 23.0 software package (IBM Corp., Armonk, NY, USA). The data were expressed as median and range or mean with standard deviation where appropriate, and compared using the Mann -Whitney $U$ test. The rates of LNM in the defined fields were shown as percentages. The risk factors for LNM were identified by univariate and multivariate logistic regression analysis. The level of significance was set at 0.05 in all tests.

\section{Results}

\section{LNM and distribution characteristics}

The incidence of LNM in this group was 14.8\% (144/975). A total of 20288 lymph nodes were removed, ranging from 11 to 49 lymph nodes per case, with a median of 19 lymph nodes per case. The positive group was compared with the negative group, and the difference was not statistically significant $(P=0.216)$. There were 359 metastatic lymph nodes, ranging from 1 to 14 per case, and the median number of metastatic lymph nodes was 2 per case.

According to the number of metastatic lymph nodes, the parauterine/obturator region accounted for the most (39.3\%, 141/359), followed by external iliac (33.1\%, 119/359), internal iliac (13.7\%, 49/359), deep inguinal (7.2\%, $26 / 359)$ and common iliac $(6.7 \%, 24 / 359)$.

According to the number of LNM locations, the number of metastases in the parauterine/obturator region was also the largest $(36.9 \%, 81 / 220)$, followed by external iliac $(33.6 \%, 74 / 220)$, internal iliac $(13.6 \%, 30 / 220)$, deep inguinal $(9.1 \%, 20 / 220)$ and common iliac $(6.8 \%, 15 / 220)$. The results of the two statistical methods are consistent.

(Table 1)

Table1 The distribution of lymph node metastasis in the patients

\begin{tabular}{|lll|}
\hline Anatomical region & \multicolumn{2}{l|}{ Lymph node metastasis } \\
\cline { 2 - 3 } & According to the number & According to occurrences \\
\hline Total & $359 \unrhd 100 \% \square$ & $220 \rrbracket 100 \% \rrbracket$ \\
common iliac & $24(6.7)$ & $15(6.8)$ \\
\hline external iliac & $119(33.1)$ & $74(33.6)$ \\
\hline internal iliac & $49(13.7)$ & $30(13.6)$ \\
\hline parauterine/obturator & $141(39.3)$ & $81(36.9)$ \\
\hline deep inguinal & $26(7.2)$ & $20(9.1)$ \\
\hline
\end{tabular}

\section{Univariate analysis of the clinical pathological parameters}

The potential clinicopathologic risk factors associated with lymphatic metastasis were investigated and analyzed. Univariate analysis showed that gravidity $>3$ times, tumor size $>4 \mathrm{~cm}$, gross type, FIGO stage, pathological type, positive LVSI, deep cervical stromal invasion (outer 1/2 invasion), PMI and UCI were all influential factors for LNM. However, there were no statistically significant differences in age, BMI, menopause, parity times, previous surgical history, tissue differentiation and vaginal margin between the two groups. (Table 2)

Table2 Univariate analysis of risk factors for lymph node metastasis 


\begin{tabular}{|c|c|c|c|c|c|}
\hline \multirow[t]{2}{*}{ Risk factors } & & \multicolumn{2}{|c|}{ Lymph node metastasis } & \multirow[t]{2}{*}{$x^{2}$} & \multirow[t]{2}{*}{$\mathrm{R}$} \\
\hline & & negative $(n=831)$ & positive $(n=144)$ & & \\
\hline \multirow[t]{2}{*}{ Age (years) } & $\leq 45$ & $417(85.5)$ & $71(14.5)$ & \multirow[t]{2}{*}{0.038} & \multirow[t]{2}{*}{0.846} \\
\hline & $\varangle 45$ & $414(85.0)$ & $73(15.0)$ & & \\
\hline \multirow[t]{2}{*}{$\mathrm{BMI}\left(\mathrm{kg} / \mathrm{m}^{2}\right)$} & $\otimes 25$ & $522(85.0)$ & $92(15.0)$ & \multirow[t]{2}{*}{0.061} & \multirow[t]{2}{*}{0.806} \\
\hline & $\geq 25$ & $309(85.6)$ & $52(14.4)$ & & \\
\hline \multirow[t]{2}{*}{ Menopausal } & \multirow[t]{2}{*}{ Premenopause Postmenopause } & $587(85.1)$ & 103(14.9) & \multirow[t]{2}{*}{0.047} & \multirow[t]{2}{*}{$0.82 \varepsilon$} \\
\hline & & $244(85.6)$ & $41(14.4)$ & & \\
\hline \multirow[t]{2}{*}{ Gravidity } & $\leq 3$ & $558(83.5)$ & $110(16.5)$ & \multirow[t]{2}{*}{4.858} & \multirow[t]{2}{*}{$0.02 \varepsilon$} \\
\hline & $\varangle 3$ & $273(88.9)$ & $34(11.1)$ & & \\
\hline \multirow[t]{2}{*}{ Parity } & $\leq 2$ & $731(85.1)$ & $128(14.9)$ & \multirow[t]{2}{*}{0.100} & \multirow[t]{2}{*}{0.752} \\
\hline & $\otimes 2$ & $100(86.2)$ & $16(13.8)$ & & \\
\hline \multirow[t]{2}{*}{ Surgical history } & No & $430(86.7)$ & $66(13.3)$ & \multirow[t]{2}{*}{1.716} & \multirow[t]{2}{*}{$0.19 c$} \\
\hline & Yes & 401(83.7) & $78(16.3)$ & & \\
\hline \multirow[t]{2}{*}{ Tumor size } & $\leq 4 \mathrm{~cm}$ & $636(88.2)$ & $85(11.8)$ & \multirow[t]{2}{*}{19.525} & \multirow{2}{*}{$\begin{array}{l}0.001 \\
0.001\end{array}$} \\
\hline & $\$ 4 \mathrm{~cm}$ & 195(76.8) & $59(23.2)$ & & \\
\hline \multirow[t]{3}{*}{ Gross type } & Exophytic & 491(88.5) & $64(11.5)$ & \multirow[t]{3}{*}{25.642} & \multirow{3}{*}{$\begin{array}{l}\square \\
0.001\end{array}$} \\
\hline & Endophytic & 272(77.9) & $77(22.1)$ & & \\
\hline & Unknown & 68(95.8) & $3(4.2)$ & & \\
\hline \multirow[t]{3}{*}{ Stage(FIG02009) } & IA & $61(95.3)$ & $3(4.7)$ & 11.883 & 0.003 \\
\hline & IB & $608(86.1)$ & $98(13.9)$ & & \\
\hline & $\| \mathrm{A}$ & $162(79.0)$ & $43(21.0)$ & & \\
\hline Histologic & $\mathrm{G} 1$ & $27(100)$ & $0(0)$ & 5.346 & 0.069 \\
\hline & G2 & $625(85.3)$ & $108(14.7)$ & & \\
\hline & G3 & 179(83.3) & $36(16.7)$ & & \\
\hline Pathologic type & Squamous cell carcinomas & $602(86.4)$ & $95(13.6)$ & 11.725 & 0.003 \\
\hline & Adenocarcinoma & $121(89.0)$ & $15(11.0)$ & & \\
\hline & adenosquamous carcinoma & 108(76.1) & $34(23.9)$ & & \\
\hline LVSI & Negative & $511(95.3)$ & $25(4.7)$ & 96.567 & a \\
\hline & Positive & $320(72.9)$ & $119(27.1)$ & & \\
\hline Stromal invasion & Inner $1 / 2$ & $487(95.3)$ & $24(4.7)$ & 86.543 & प0 \\
\hline & Outer $1 / 2$ & $344(74.1)$ & $120(25.9)$ & & \\
\hline
\end{tabular}




\begin{tabular}{|llllll|} 
Vaginal margin & Negative & $821(85.3)$ & $141(14.7)$ & 0.208 & 0.648 \\
& Positive & $10(76.9)$ & $3(23.1)$ & & \\
PMI & Absent & $812(86.2)$ & $130(13.8)$ & 18.540 & $\square$ \\
& Present & $19(57.6)$ & $14(42.4)$ & & 0.001 \\
UCI & No & $765(87.3)$ & $111(12.7)$ & 30.933 & 0 \\
& Endometrial invasion & $13(86.7)$ & $2(13.3)$ & & 0.001 \\
& Myometrial invasion $₫ 50 \%$ & $43(68.3)$ & $20(31.7)$ & & \\
& Myometrial invasion $\geq 50 \%$ & $10(47.6)$ & $11(52.4)$ & & \\
& & & \\
\hline
\end{tabular}

$\mathrm{BMI}=$ body mass index, $\mathrm{LVSI}=$ lymphovascular space involvement, $\mathrm{PMI}=$ parametrial invasion, $\mathrm{UCl}=$ uterine corpus invasion.

\section{Multivariate logistic regression analysis of patients with LNM}

To further confirm the identified risk factors, the multivariate logistic regression analysis were performed. LNM was only associated with tumor size $\otimes 4 \mathrm{~cm}$, positive LVSI, cervical staomal outer $1 / 2$ invasion, and deep UCI (myometrial invasion $\geq 50 \%$ ). (Table 3 )

Table3 Multivariate analysis of risk factors for lymph node metastasis

\begin{tabular}{|c|c|c|c|c|}
\hline Variables & Comparison & OR & $95 \% \mathrm{Cl}$ & $\mathrm{P}$ \\
\hline Tumor size & $\varangle 4 \mathrm{~cm}$ vs. $\leq 4 \mathrm{~cm}$ & 2.253 & $1.486-3.416$ & $\$ 0.001$ \\
\hline LVSI & Positive vs. Negative & 5.353 & $3.303-8.676$ & $\otimes 0.001$ \\
\hline Stromal invasion & Outer $1 / 2$ vs. Inner $1 / 2$ & 3.461 & $2.106-5.688$ & $\otimes 0.001$ \\
\hline \multirow[t]{3}{*}{$\mathrm{UCl}$} & Endometrial invasion vs. No & 0.554 & $0.111-2.767$ & 0.471 \\
\hline & Myometrial invasion $₫ 50 \%$ vs. No & 1.821 & $0.976-3.398$ & 0.060 \\
\hline & Myometrial invasion $\geq 50 \%$ vs. No & 3.529 & $1.321-9.427$ & 0.012 \\
\hline
\end{tabular}

$\mathrm{Cl}$ = confidence interval, LVSI = lymphovascular space involvement, $\mathrm{UCI}=$ uterine corpus invasion.

\section{Discussion}

\section{Main findings}

Our data showed that the incidence of LNM in 975 cervical cancer patients was $14.8 \%$, and the metastasis rates were $4.7 \%$ in stage IA, $13.9 \%$ in stage IB, and $21.0 \%$ in stage IIA. Although clinical stage is not an independent risk factor for LNM, it can be seen that the rate of LNM increases with the increase of clinical stage, and this result is consistent with previous reports $[15,16]$. We further analyzed the partitions of metastatic lymph nodes, and the conclusions were consistent according to the number and frequency of metastatic lymph nodes. The results showed that the parauterine/obturator regions were the most easily affected anatomical partitions. Similar reports also remind us to pay attention to LNM excision of the parauterine and obturator regions[16, 17]. 
It can be seen that among the four independent risk factors, "positive LVSI" is the factor with the highest OR value (table 3), and similar reports have been reported in the past $[18,19]$. Therefore, we should pay special attention to preoperative pathological with "LVSI positive", even if imaging examination did not suggest metastasis. Then we further analyzed the relationship between preoperative pathology and LNM, and found that among all patients (975), 416 cases were positive LVSI as indicated by preoperative pathology, while 107 cases were confirmed for LNM by postoperative pathology, accounting for 74.3\% (107/144) of all LNM patients. In addition, it can be seen that 3 patients with stage IA had LNM (table 2). After careful review of clinical data, preoperative biopsy pathology showed positive LVSI in all 3 patients. It is suggested that attention should be paid to the "positive LVSI" result of preoperative biopsy, and systematic lymphadenectomy is required even in stage IA [20].

Deep inguinal lymph node resection is an independent risk factor for lymphedema of lower extremity [21]. The removal of deep inguinal lymph nodes has been controversial for a long time. In our study, there was a certain rate of deep inguinal LNM, and a total of 20 patients had deep inguinal LNM, among which 18 patients (18/20) were positive LVSI. Therefore, for people with independent risk factors, especially positive LVSI, systematic resection of lymph nodes, including deep inguinal lymph nodes, should be recommended.

In the past, we did not pay enough attention to $\mathrm{UCl}$, and believe that it did not affect staging. In this study, 99 patients had $\mathrm{UCl}$, and the LNM rate of patients with endometrial, myometrial invasion $<50 \%$ and myometrial invasion $\geq 50 \%$ was $13.3 \%, 31.7 \%$ and $52.4 \%$ respectively. Among them, only uterine deep myometrial invasion was an independent risk factor affecting LNM. Therefore, we remind that great attention must be paid to UCl of cervical cancer, especially to the "depth of UCl". It is recommended to strengthen preoperative examination and evaluation for these patients, and complete lymph node resection may also be needed. In addition, studies have shown that LNM is also an independent risk factor for UCI[22], and uterine deep myometrial invasion is an independent risk factor for poor prognosis of cervical cancer patients[23,24]. The presence of UCI should be considered as a poor prognostic factor in the diagnosis and treatment protocol, and systematic chemotherapy or prophylactic irradiation of the para-aortic lymph node region may be considered as an option due to its increased risk of para-aortic LNM [25].

In conclusion, it is recommended to strengthen the evaluation of LNM if there are risk factors such as tumor size> 4cm, positive LVSI, deep cervical stromal invasion or deep UCl. Currently, imaging examinations, such as magnetic resonance imaging (MRI) or computed tomography (CT), are commonly used to assess LNM. But relevant articles show that diffusion weighted-MRI (DW-MRI) has the highest sensitivity and positron emission tomography-CT (PET-CT) has the highest specificity [15, 26-28]. Therefore, the intensity of preoperative imaging examination should be strengthened and attention should be paid to the treatment when the above risk factors occur, and it is suggested to consider systematic complete lymph node resection.

\section{Limitations}

Although some studies support that LNM is related to tissue differentiation [29], this study did not find this result. In addition, Li et al. [30] pointed out that endogenous type was an independent risk factor for PMI. Our study analyzed the relationship between LNM and gross type, and the results showed that the difference was statistically significant in univariate analysis, but it was not an independent risk factor. The reason may be related to the large span of FIGO stage in this study and the vague gross type of some early patients. Since this study is a single-center and retrospective study, it needs a multi-center, large-sample and prospective study to provide a more valuable reference for clinical practice. 


\section{Significance}

In recent years, there have been many studies on sentinel lymph node[31-33], and relevant articles have pointed out that sentinel lymph node mapping has a good detection rate for early-stage cervical cancer tumors [34,35], but in early-stage cervical cancer, overall detection rate of sentinel lymph node mapping is elevated while bilateral detection rate is lower[36].

Therefore, according to the principle of individualized treatment, for early-stage cervical cancer without lymph node enlargement and metastasis in imaging examination, can it be classified as low-risk or high-risk according to the risk of LNM? Meanwhile, for early-stage cervical cancer, can systematic lymph node dissection be performed only in high-risk patients? Sentinel lymph node mapping in low-risk patients? In conclusion, for early-stage cervical cancer, attention should be paid to the evaluation of LNM, and it is urgent to form evaluation criteria and optimize evaluation methods as soon as possible.

\section{Declarations}

Author contributions LC is first author, LC designed the study and wrote the paper. WK supervised and revised the paper. JL, CH and TL conducted the analyse. LC, BJ and DS collected the data. All authors have read and approved the final manuscript.

\section{Compliance with ethical standards}

Conflict of interest The authors declare that there is no conflict of interest regarding the publication of this article.

Ethical approval The study used previously collected/existing clinical data and medical records; therefore, the need for informed consent was waived. The study was conducted in accordance with the 1964 Declaration of Helsinki and its subsequent revisions.

\section{References}

1. Sharma S, Deep A, Sharma AK (2020) Current Treatment for Cervical Cancer: An Update. Anticancer Agents Med Chem 20: 1768-1779. https://doi.org/10.2174/1871520620666200224093301

2. Sung H, Ferlay J, Siegel R et al (2021) Global Cancer Statistics 2020: GLOBOCAN Estimates of Incidence and Mortality Worldwide for 36 Cancers in 185 Countries. CA Cancer J Clin 71: 209-249. https://doi.org/10.3322/caac.21660

3. Hosaka M, Watari H, Mitamura T et al (2010) Survival and prognosticators of node-positive cervical cancer patients treated with radical hysterectomy and systematic lymphadenectomy. Int J Clin Oncol 16: 33-38. https://doi.org/10.1007/s10147-010-0123-0

4. Balaya V, Guani B, Bonsang-Kitzis H et al (2020) Sentinel lymph node biopsy in early-stage cervical cancer: current state of art. Bull Cancer 107: 696-706. https://doi.org/10.1016/j.bulcan.2019.06.011

5. Bhatla N, Berek JS, Cuello Fredes M et al (2019) Revised FIGO staging for carcinoma of the cervix uteri. Int J Gynaecol Obstet 145: 129-135. https://doi.org/10.1002/ijgo.12749

6. Jeong SY, Park H, Kim MS et al (2020) Pretreatment Lymph Node Metastasis as a Prognostic Significance in Cervical Cancer: Comparison between Disease Status. Cancer Res Treat 52: 516-523.

https://doi.org/10.4143/crt.2019.328 
7. de Gregorio A, Widschwendter P, Ebner F et al (2020) Influence of the New FIGO Classification for Cervical Cancer on Patient Survival: A Retrospective Analysis of 265 Histologically Confirmed Cases with FIGO Stages IA to IIB. Oncology 98: 91-97. https://doi.org/10.1159/000503149

8. Schmeler KM, Pareja R, Lopez Blanco A et al (2021) ConCerv: a prospective trial of conservative surgery for low-risk early-stage cervical cancer. Int J Gynecol Cancer 31: 1317-1325. https://doi.org/10.1136/ijgc-2021002921

9. Wu C, Li L, Xiao X et al (2019) Risk Factors of Regional Lymph Node Metastasis in Patients with Cervical Cancer. Open Life Sci 14: 208-213. https://doi.org/10.1515/biol-2019-0023

10. Liu Y, Zhao LJ, Li MZ et al (2015) The number of positive pelvic lymph nodes and multiple groups of pelvic lymph node metastasis influence prognosis in stage IA-IIB cervical squamous cell carcinoma. Chin Med J (Engl) 128: 2084-2089. https://doi.org/10.4103/0366-6999.161372

11. Aslan K, Haberal A, Akıllı $\mathrm{H}$ et al (2021) Prognostic value of the number of the metastatic lymph nodes in locally early-stage cervical cancer: squamous cell carcinoma versus non-squamous cell carcinoma. Arch Gynecol Obstet 304: 1279-1289. https://doi.org/10.1007/s00404-021-06030-w

12. Zhou J, Ran J, He ZY et al (2015) Tailoring Pelvic Lymphadenectomy for Patients with Stage IA2, IB1, and IIA1 Uterine Cervical Cancer. J Cancer 6: 377-381. https://doi.org/10.7150/ jca.10968

13. Wang Y, Yao T, Yu J et al (2016) Can pelvic lymphadenectomy be omitted in patients with stage IA2, IB1, and IIA1 squamous cell cervical cancer? Springerplus 5: 1262-1268. https://doi.org/10.1186/s40064-016-2927-5

14. Togami S, Kamio M, Yanazume S et al (2014) Can pelvic lymphadenectomy be omitted in stage IA2 to IIB uterine cervical cancer? Internat J Gynecol Cancer 24: 1072-1076. https://doi.org/10.1097/igc.00000 00000000163

15. Olthof EP, van der Aa MA, Adam JA et al (2021) The role of lymph nodes in cervical cancer: incidence and identification of lymph node metastases-a literature review. Int J Clin Oncol 26: 1600-1610. https://doi.org/10.1007/s10147-021-01980-2

16. Nanthamongkolkul K, Hanprasertpong J (2018) Predictive Factors of Pelvic Lymph Node Metastasis in EarlyStage Cervical Cancer. Oncol Res Treat 41: 194-198. https://doi.org/10.1159/000485840

17. Cai J, He X, Wang H et al (2021) Topographic distribution of lymph node metastasis in patients with stage IB1 cervical cancer: an analysis of 8314 lymph nodes. Radiat Oncol 16: 54-63. https://doi.org/10.1186/s13014021-01781-x

18. Zhao J, Cai J, Wang H et al (2021) Region-specific Risk Factors for Pelvic Lymph Node Metastasis in Patients with Stage IB1 Cervical Cancer. J Cancer 12: 2624-2632. https://doi.org/10.7150/jca.53215

19. Balaya V, Guani B, Magaud L et al (2020) Validation of the 2018 FIGO Classification for Cervical Cancer: Lymphovascular Space Invasion Should Be Considered in IB1 Stage. Cancers 12: 3554-3567.

https://doi.org/10.3390/cancers12123554

20. Margolis B, Cagle-Colon K, Chen L et al (2020) Prognostic significance of lymphovascular space invasion for stage IA1 and IA2 cervical cancer. Int J Gynecol Cancer 30: 735-743. https://doi.org/10.1136/ijgc-2019-0008

21. Ohba $Y$, Todo $Y$, Kobayashi $N$ et al (2011) Risk factors for lower-limb lymphedema after surgery for cervical cancer. Int J Clin Oncol 16: 238-243. https://doi.org/10.1007/s10147-010-0171-5

22. Li W, He F, Liu P et al (2021) Uterine corpus invasion in cervical cancer: a multicenter retrospective case-control study. Arch Gynecol Obstet 303: 777-785. https://doi.org/10.1007/s00404-021-05968-1 
23. He F, Li W, Liu P et al (2020) Influence of uterine corpus invasion on prognosis in stage IA2-IIB cervical cancer: A multicenter retrospective cohort study. Gynecol oncol 158: 273-281.

https://doi.org/10.1016/j.ygyno.2020.05.005

24. Matsuo K, Machida H, Blake EA et al (2017) Significance of uterine corpus tumor invasion in early-stage cervical cancer. Eur J Surg Oncol 43: 725-734. https://doi.org/10.1016/j.ejso.2017.01.017

25. Turan T, Kimyon Comert G, Boyraz G et al (2021) What is the impact of corpus uterine invasion on oncologic outcomes in surgically treated cervical cancer? J Obstet Gynaecol Res 47: 3634-3643.

https://doi.org/10.1111/jog.14953

26. Woo S, Atun R, Ward ZJ et al (2020) Diagnostic performance of conventional and advanced imaging modalities for assessing newly diagnosed cervical cancer: systematic review and meta-analysis. Eur Radiol 30: 5560-5577. https://doi.org/10.1007/s00330-020-06909-3

27. Haldorsen IS, Lura N, Blaakær J et al (2019) What Is the Role of Imaging at Primary Diagnostic Work-Up in Uterine Cervical Cancer? Curr Oncol Rep 21: 77-91. https://doi.org/10.1007/s11912-019-0824-0

28. Wang T, Gao T, Yang J et al (2019) Preoperative prediction of pelvic lymph nodes metastasis in early-stage cervical cancer using radiomics nomogram developed based on T2-weighted MRI and diffusion-weighted imaging. Eur J Radiol 114: 128-135. https://doi.org/10.1016/j.ejrad.2019.01.003

29. Canaz E, Ozyurek ES, Erdem B et al (2017) Preoperatively Assessable Clinical and Pathological Risk Factors for Parametrial Involvement in Surgically Treated FIGO Stage IB-IIA Cervical Cancer. Int J Gynecol Cancer 27: 1722-1728. https://doi.org/10.1097/IGC.0000000000001060

30. Li D, Cai J, Kuang Y et al (2012) Surgical-pathologic risk factors of pelvic lymph node metastasis in stage lb1Ilb cervical cancer. Acta Obstet Gynecol Scand 91: 802-809. https://doi.org/10.1111/j.16000412.2012.01415.x

31. Wu Y, Li Z, Wu H et al (2013) Sentinel lymph node biopsy in cervical cancer: a meta-analysis.Mol Clin Oncol 1:1025-1030. https://doi.org/10.3892/mco.2013.168

32. Ulain Q, Han L, Wu Q et al (2018) Indocyanine green can stand alone in detecting sentinel lymph nodes in cervical cancer. J Int Med Res 46: 4885-4897. https://doi.org/10.1177/0300060518803041

33. Zhang X, Bao B, Wang S et al (2021) Sentinel lymph node biopsy in early stage cervical cancer: A metaanalysis. Cancer Med 10: 2590-2600. https://doi.org/10.1002/cam4.3645

34. Wang XJ, Fang F, Li YF (2015) Sentinel-lymph-node procedures in early stage cervical cancer: a systematic review and meta-analysis. Med Oncol 32: 1-12. https://doi.org/10.1007/s12032-014-0385-x

35. Kadkhodayan S, Hasanzadeh M, Treglia G et al (2015) Sentinel node biopsy for lymph nodal staging of uterine cervix cancer: a systematic review and meta-analysis of the pertinent literature. Eur J Surg Oncol 41: 1-20. https://doi.org/10.1016/j.ejso.2014.09.010

36. Wang L, Liu S, Xu T et al (2021) Sentinel lymph node mapping in early-stage cervical cancer: Meta-analysis. Medicine 100: e27035. https://doi.org/10.1097/MD.0000000000027035 